\title{
The effect of patient positioning during radiographs on the resulting Cobb angle measurements
}

\author{
M Siljander ${ }^{1 *}$, P Knott ${ }^{1}$, S Thompson ${ }^{2}$, S Mardjetko $^{2}$ \\ From 8th International Conference on Conservative Management of Spinal Deformities and SOSORT 2011 \\ Annual Meeting \\ Barcelona, Spain. 19-21 May 2011
}

\section{Background}

Standing spinal radiographs have been the primary method of spinal deformity evaluation in patients with scoliosis. During periods of patient surveillance, the clinician compares radiographs over a period of time to assess the progression of the deformity [1-3]. One of the potential problems in comparing one radiograph to another is difference in positioning [4-6]. The goal of this study is to quantify the effect of trunk rotation on Cobb angle measurements, and provide an algorithm to describe this relationship.

\section{Material and methods}

CT scans of three patients with Adolescent Idiopathic Scoliosis were used retrospectively. Three-dimensional reconstructions of the images were created by CT scan software. Cobb angles were drawn for scoliosis curves in the anterior plane. The 3-D image was then rotated two degrees to the right, and Cobb angle measurements were repeated. This procedure was repeated through 14 degrees of right rotation, and then subsequently through 14 degrees of left rotation.

\section{Results}

The effect of trunk rotation on Cobb angle measurements is directly related to the location of the scoliosis curves, the magnitude of those curves, and the magnitude of lumbar lordosis and thoracic kyphosis. In general however, a two degree rotation of the patient's trunk while positioning results in a one degree change in the measured Cobb angle (in patients with larger scoliosis curves, and in the first six degrees of trunk rotation).

\section{Conclusions}

Patient positioning can have a significant effect on the calculation of scoliosis measurements, and this needs to be considered when evaluating the progression of spinal deformity.

\section{Author details \\ 'Rosalind Franklin University North Chicago, USA. ${ }^{2}$ Illinois Bone and Joint Institute Morton Grove, USA.}

Published: 27 January 2012

\section{References}

1. Lonstein JE, Carlson JM: The prediction of curve progression in untreated idiopathic scoliosis during growth. J Bone Joint Surgery [Am] 1984, 66:1061-71.

2. Screening for adolescent idiopathic scoliosis. Policy statement. US Preventative Services Task Force. JAMA 1993, 269:2664-6.

3. Asher MA, Burton DC: Adolescent idiopathic scoliosis: natural history and long term treatment effects. Scoliosis 2006, 1:2, doi: 10.1186/1748-1761-1-2.

4. Cummings RJ, Loveless EA, Campbell J, et al: Interobserver reliability and intraobserver reproducibility of the system of King et al. for the classification of adolescent idiopathic scoliosis. J Bone Joint Surg Am 1998, 80(8):1107-11.

5. Lenke LG, Betz RR, Bridwell KH, et al: Intraobserver and interobserver reliability of the classification of thoracic adolescent idiopathic scoliosis. J Bone Joint Surg Am 1998, 80(8):1097-106.

6. Morrissy RT, Goldsmith GS, Hall EC, et al: Measurement of the Cobb angle on radiographs of patients who have scoliosis. Evaluation of intrinsic error. J Bone Joint Surg Am 1990, 72(3):320-7.

doi:10.1186/1748-7161-7-S1-012

Cite this article as: Siljander et al:: The effect of patient positioning during radiographs on the resulting Cobb angle measurements. Scoliosis 2012 7(Suppl 1):012.

${ }^{1}$ Rosalind Franklin University North Chicago, USA

Full list of author information is available at the end of the article

(c) 2012 Siljander et al; licensee BioMed Central Ltd. This is an open access article distributed under the terms of the Creative Commons 